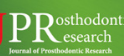 \\ Journal of Prosthodontic Research
}

\author{
Official Journal of Japan Prosthodontic Society
}

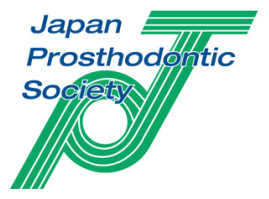

Original article

\section{Newly designed flat surface artificial tongue system for speech improvement in glossectomy patients: A preliminary study}

\author{
Hiroaki Nagatsuka, Shigehisa Kawakami, Miho Kuwahara, Chie Kubota, Naoki Kodama*, Shogo Minagi

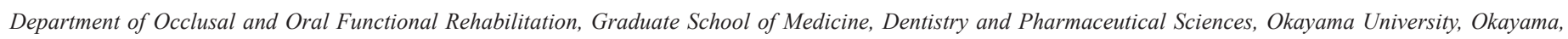 \\ Japan
}

\begin{abstract}
Purpose: To design an efficient tongue prosthesis with reproducibility and to objectively evaluate improvement in speech function. Methods: A silicon anatomical artificial tongue (AT) and a flat surface artificial tongue system (FTS) were used in our study. Twenty healthy participants (10 males and 10 females, $26.3 \pm 1.8$ years) were fitted with a tongue movement suppression appliance (TSA) that fit the dental arch to simulate the glossectomy condition. TSA, TSA + FTS, and TSA + AT simulated the state of glossectomy patients without artificial tongue, with normal artificial tongue, and newly designed artificial tongue, respectively. Three speech intelligibility tests were performed for each of the following conditions: pronouncing 100 Japanese monosyllables, 40 Japanese words, and reading a short story. One-way ANOVA, Wilcoxon signed-rank test, and Tukey-Kramer post-hoc test were used for statistical analyses.

Results: Significant differences were observed for 100 Japanese monosyllables and 40 Japanese words between the TSA + FTS, TSA, and TSA + AT conditions (p $<0.05)$. Regarding the speech intelligibility test for reading a short story, the TSA + FTS condition resulted in a significantly higher speech intelligibility than the TSA and TSA + AT conditions $(\mathrm{p}<0.05)$.

Conclusions: A flat surface artificial tongue system contributed to the improvement in speech function. This structure can be easily used in cases where conventional artificial tongue are applicable, regardless of variation in the oral condition; thus, making it a widely applicable treatment option for glossectomy patients.
\end{abstract}

Keywords: Artificial tongue, Speech intelligibility, Glossectomy, Tongue suppression, Tongue tumor

\section{Introduction}

Oral and pharyngeal cancers account for approximately $3 \%$ of all malignant tumors in men and $2 \%$ of all malignant tumors in women [1]. For oral cancer patients, glossectomy is a drastic treatment option that reduces their quality of life $[2,3,4,5,6]$.

Tongue reconstructive surgery and rehabilitation are effective options for functional recovery following glossectomy. Successful reconstructive surgery enables patients to regain speech, although it is often difficult to predict its effect on functional improvement; however, tongue reconstruction surgery is not possible in all glossectomy patients, including those with tumor recurrence. A previous systematic review and metaanalysis study [7] has shown that prosthetic rehabilitation in conjunction with surgical reconstruction could be one of the best strategies to improve voice, speech, and swallowing function. Tongue prosthesis is used as a treatment option if tongue reconstruction surgery is not effective $[8,9]$. Speaking and swallowing are everyday functions in which the tongue plays a crucial role, and it is essential for communication, and it has a huge impact on the quality of life (QOL) of oral cancer survivors.

Tongue prostheses have been reported as a prosthetic rehabilitation

\footnotetext{
* Corresponding author at: Department of Occlusal and Oral Functional Rehabilitation, Graduate School of Medicine, Dentistry and Pharmaceutical Sciences, Okayama University, 2-5-1 Shikata-cho, Okayama, 700-8525, Japan.

E-mail address: naoki-k@md.okayama-u.ac.jp (N. Kodama).
}

method for tongue resection $[10,11,12]$. However, most case reports consist of a small number of patients; further, there are no previous reports on morphological considerations for the design of a universal tongue prosthesis. There is a wide range of variations in the oral cavity of glossectomy patients. This variation makes it difficult to standardize the form of the artificial tongue. Therefore, dentists must fabricate each artificial tongue keeping in mind the individual circumstances, which might be a difficult task without proper knowledge. This could be due to the low probability of successful prosthetic rehabilitation after tongue resection. An efficient solution would be to design an artificial tongue with a reasonably simplified structure, which can be implemented even in medical institutions with only a few such cases.

For the past five years, we have operated an outpatient clinic in our facility, which specializes in prosthetic treatment for patients with tongue resection [13]. As pointed out in a review by Balasubramaniam et al., a major problem not addressed by previous case reports on tongue prosthesis is the variation in oral morphology [14]. When the speech function is recovered by a trial and error process depending on the characteristics of each case, it becomes difficult to derive any generalization from such cases. Furthermore, since the artificial tongue is applicable only to a small number of patients in clinics, it is difficult for prosthodontists to treat such patients because of a lack of experience. Thus, we have recently adopted an effective and simplified tongue prosthesis design for speech rehabilitation in our clinic.

The purpose of this study was to design an efficient tongue prosthesis with reproducibility and to objectively evaluate the improvement in articulatory function. The research hypothesis was that there is no difference in speech intelligibility between the simplified design of a tongue prosthesis and a conventional artificial tongue. 


\section{Materials and methods}

Twenty subjects ( 10 males and 10 females, $26.3 \pm 1.8$ years) who were either dentists or undergraduate students at the Okayama University Hospital, participated in this study. Informed consent was obtained from all participants.

All participants were native Japanese speakers and did not have disarthria or auditory disorders subjectively and/or objectively. To simulate the oral condition of glossectomy patients, we built a removable tongue movement suppression appliance (TSA) and two artificial tongues for all participants. One was a silicon anatomical artificial tongue (AT), and the other was a flat surface artificial tongue system (FTS). These devices were fabricated using the following working model: Impressions of the upper and lower dental arches of each participant were first taken using a readymade tray and an alginate impression material, and dental stone was then poured into the impressions to produce working casts.

\subsection{Fabrication of the TSA}

The TSA consisted of a component that fit the dental arch to secure the device and a flat tongue suppression plate to suppress tongue movement (Fig. 1). A thermoelastic plate (0.5 mm, DURAN+, SCHEU DENTAL) was fixed on the dental arch, and a flat plate $(1.5 \mathrm{~mm}$, DURAN + , SCHEU DENTAL) was adhered parallel to the occlusal plane. This vacuum-formed TSA required no occlusal adjustment except at the margin of this device so that participants could feel comfortable with it.

\subsection{Fabrication of the $A T$}

AT is a silicon-made artificial tongue with the shape of a standard tongue model. The AT was fabricated using a denture soft liner (SOFRELINER Super Soft, Tokuyama Dental, Tokyo) in the shape of a standard tongue model with a thickness of $20 \mathrm{~mm}$, a width of $30 \mathrm{~mm}$, and a length of 40 mm (Z-1, Nissin Dental Nissin Products Inc.). It was fitted to the TSA using a metal clip (Fig. 2a and 2b). An equally sized AT was applied to all participants, regardless of the difference in the shape of the palate.

\subsection{Fabrication of the FTS}

The FTS consists of a silicon-made flat surface artificial tongue and a flat surface palatal augmentation prosthesis (PAP). The FTS consists of a flat PAP applied to the upper jaw and an artificial tongue that fit the flat surface of the PAP (Fig. 3). The PAP was fabricated using orthodontic resin (Orthopallet, Shofu, Kyoto), which had a wire clasp that could be fastened to the last molar tooth. The posterior border of the PAP corresponded to the distal portion of the first molar on both sides, and its height corresponded to the height of the cervical margin of the upper dental arch. The FTS was fabricated using a soft denture liner (SOFRELINER Super Soft, Tokuyama Dental, Tokyo) to coincide with the maximum intercuspal position after insertion of the TSA and the flat PAP, which was built to fill the space between the TSA and the flat PAP. The distance from the occlusal surface to the flat plate was approximately $1 \mathrm{~mm}$, and the distance was not regulated. As noticed in 2.1. Fabrication of the TSA, occlusal adjustment was not necessary in all participants. The red-colored flat area in Fig. 3b is in full contact with the flat PAP. An artificial tongue was attached to the TSA using a metal clip.

\subsection{Speech intelligibility test}

The speech recording was performed in a quiet room where the participants were instructed to sit in an upright position while wearing noise-cancelling headphones. An electrostatic microphone (PG-27LC, Shure Incorporated) was placed in front of the participants at a distance of $15 \mathrm{~cm}$. During both the practice and recording sessions, the participants spoke while listening to their voice in real time with a headphone. The voice was recorded at a loudness of $60 \mathrm{~dB}$ through an audio interface (OCTA-CAPTURE UA-1010), as shown in Fig.4. The recordings were made in the following sequence: first with TSA, followed by FTS/AT a

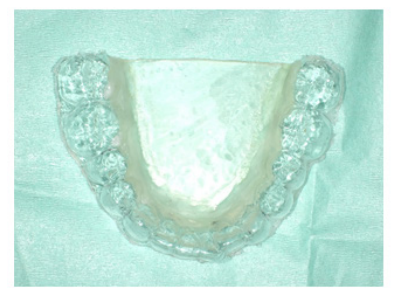

b

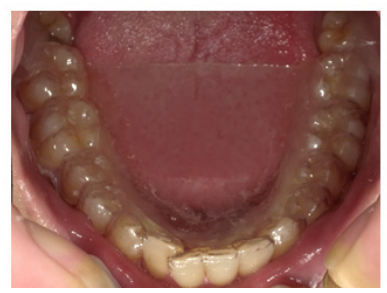

Fig. 1. Tongue movement suppression appliance. a: occlusal view of tongue movement suppression appliance (TSA); b: wearing TSA; c: schematic diagram of TSA in position. a

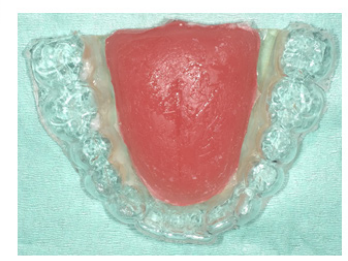

b

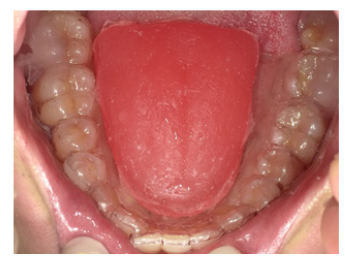

C

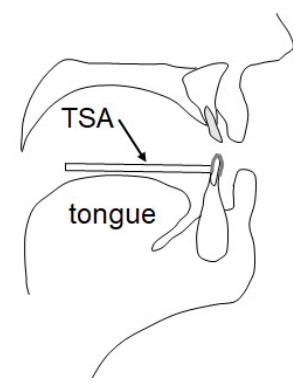

Fig. 2. Artificial tongue. a: tongue movement suppression appliance with anatomical tongue prosthesis (TSA + AT); b: wearing TSA + AT; c: schematic diagram of TSA + AT. a

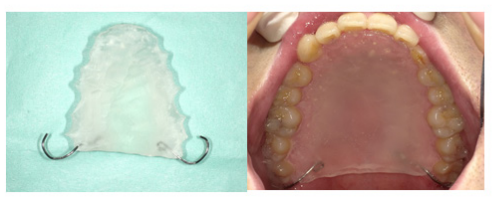

b

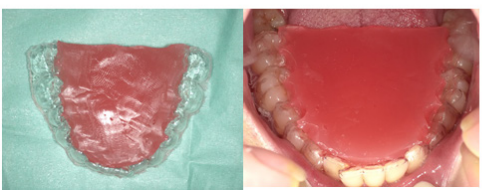

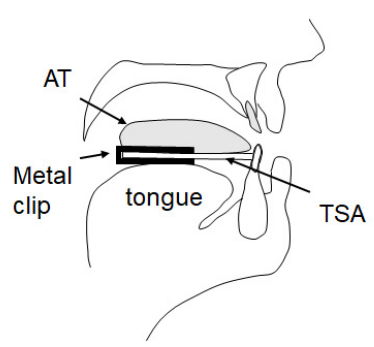

Fig. 3. Flat surface tongue prosthesis. a: flat palatal augmentation prosthesis; b: tongue movement suppression appliance with flat surface tongue prosthesis (TSA + FTS); c: schematic diagram of TSA + FTS.
C

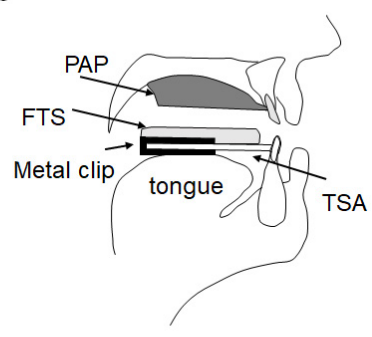




\section{Recording Sessions}

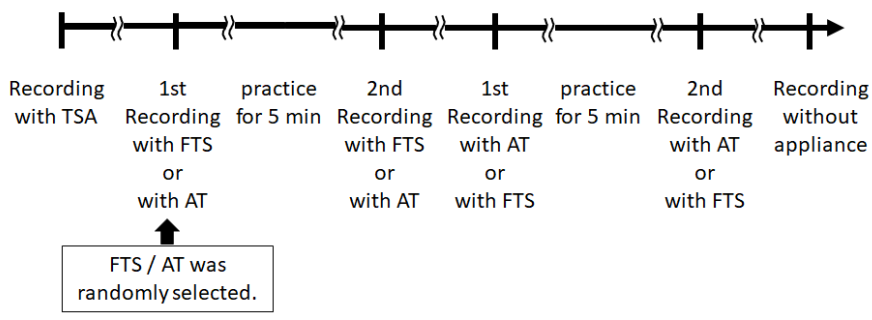

Fig. 4. Recording sessions. Each recording session included speaking 100 Japanese monosyllables, 40 Japanese words, and reading a short story titled "The North Wind and the Sun.".

(selected randomly), and finally without any appliance. Participants were allowed to rest between each recording session. Participants were asked to attach and detach all the devices throughout the recording session. Prior to the second recording with FTS/AT, the participants practiced voluntary speech for five minutes. Although only TSA and no appliance were measured only once, FTS/AT was measured twice. With regard to FTS/AT, the mean values of the first and second measured results were used as representative values. The recorded audio was evaluated by five untrained examiners who did not have any auditory abnormalities. None of the examiners were informed about the condition of the participants in advance.

\subsubsection{Speech intelligibility test with 100 Japanese monosyllables}

The test was performed in accordance with the method formulated by Umino et al. [14]. Hundred monosyllabic Japanese syllables were randomly arranged on the test table, and participants were instructed by the examiner to pronounce the monosyllables sequentially, which was then recorded. Five examiners were asked to hear the audio that was recorded and write it down exactly as it was heard. The mean value of the percentage of words correctly heard was regarded as the specch intelligibility score for each recording.

\subsubsection{Speech intelligibility test with 40 Japanese words}

The speech intelligibility test using 40 Japanese words was carried out in accordance with the method described by Johansson et al. [15], wherein participants were instructed to read a randomly arranged list of words. Speech intelligibility was calculated in accordance with the method described in Section 2.4.1. Speech intelligibility test with 100 Japanese monosyllables.

\subsubsection{Speech intelligibility test while reading a short story}

Participants were instructed to read a short story titled "The North Wind and the Sun" in Japanese, which consisted of 225 speech sounds at their own speed. The listeners evaluated each participant's reading on a scale of 1 to 5 based on the following criteria: 1. intelligible; 2 . occasionally intelligible; 3 . intelligible if the topic was familiar; 4 . occasionally unintelligible and 5. unintelligible. The mean speech intelligibility score marked by the five listeners was used to calculate the test results [16].

\subsection{Statistical analyses}

The significance level was set at $5 \%$ for statistical analyses. Regarding the results of the speech intelligibility test for 100 Japanese monosyllables and 40 Japanese words, one-way repeated measures analysis of variance (ANOVA) and Tukey-Kramer test as a post hoc test were used, while the Wilcoxon signed-rank test was used for the results of the test that involved reading a short story.

The study protocol was approved by the ethics committee of Okayama University (No. 1803-001). Written informed consent was obtained from all volunteers participating in this study.

\section{Results}

There was no significant sex difference among the conditions in speech intelligibility, except for with TSA ( $\mathrm{p}=0.0447)$. There was no significant difference between 1 st and 2 nd recordings in speech intelligibility with $\mathrm{TSA}+\mathrm{AT}(\mathrm{p}=0.4209)$. There was a significant difference between 1st and 2nd recordings in speech intelligibility with TSA+FTS ( $\mathrm{p}=0.001)$, with 2 nd recording being better than 1 st recording .

The speech intelligibility results of the test with 100 Japanese monosyllables for the conditions without appliance, with TSA, TSA + AT, and TSA + FTS were $90.2 \pm 5.2 \%, 36.8 \pm 8.0 \%, 53.4 \pm 9.8 \%$, and $64.5 \pm$ $8.2 \%$, respectively (Fig. 5a). The speech intelligibility results of the test with 40 Japanese words for the conditions without appliance, with TSA, with TSA + AT, and with TSA + FTS were $98.6 \pm 1.0 \%, 74.2 \pm 5.6 \%, 85.4$ $\pm 3.9 \%$, and $89.5 \pm 3.8 \%$, respectively (Fig. $5 \mathrm{~b}$ ). Concerning the test that involved reading a short story called "The North Wind and the Sun," the results for the conditions without appliance, with TSA, with TSA + AT, and with TSA + FTS were $1.0 \pm 0.0,3.4 \pm 0.5,3.2 \pm 0.5$, and $2.8 \pm 0.3$, respectively (Fig. 5c). As shown in Fig. 5, TSA + FTS showed significantly higher intelligibility than both TSA and TSA + AT $(\mathrm{p}<0.05)$ in all tests.

\section{Discussion}

The difficulty in restoring total and subtotall glossectomy patients' speech prompted us to seek a universal design and concept for good oral appliance for speech. Based on previous case reports and literature, we fabricated an anatomical tongue prosthesis. However, tooth axis inclination to the lingual side, which could often be caused by the lack of tongue pressure, tended to allow air to escape between the anatomical artificial tongue and the cervical area of the premolars and molars, which hampered the effective sealing during exhalation to produce clear dental and postalveolar plosives, and clear alveolar fricatives. To overcome the difficulties caused by the escape of air in the vicinity of the posterior teeth, we employed conventional PAP with an anatomical artificial tongue. However, unlike the movable muscular tongue, it is usually difficult or impossible for glossectomy patients to make good air seal in the vicinity of posterior teeth and simultaneously make a good articulation point with bulky untransformative artificial tongue and PAP. After these cases and trial and error, flat surfaces on the upper and lower dental arch that fit differently by purposeful mandibular movements of the patient was revealed to be easy and effective in controlling exhalation during speech production. This study aimed to objectively examine the effect of a flat surface artificial tongue design, which was clinically supported by our glossectomy patients. Figure 6 shows an example of an artificial tongue prosthesis with narrower effective flat surfaces with an additional protuberance dorsally to support better resonance.

To the best of our knowledge, this is the first study to evaluate the efficacy of a flat-surface artificial tongue system. One of the merits of this system lies in the fact that there is little or no need to adjust the appliance. In this study, none of the participants required adjustment for FTS+TSA. Despite no adjustment, TSA + FTS could improve speech intelligibility compared to TSA or TSA + AT. The structure of the FTS made it possible to broaden the palate-tongue contact area, leading to increased contact area between the palate and tongue during speaking, contributing to speech improvement. In addition, considering the difference between 1 st and 2 nd recordings for speech intelligibility with TSA + FTS, this prosthesis might have an effect of practice on speech production compared to ordinary artificial tongue.

However, the newly designed flat-surface artificial tongue has some disadvantages. One problem is the amount of maximum mouth opening in patients. It is difficult to take impression for an FTS in patients with Trismus as cast models of the maxilla and mandible are necessary for FTS fabrication. The severe limitation of mouth opening makes it impossible to fabricate an FTS. The second problem is the retention of saliva in the oral cavity. This is the same problem as seen in patients wearing mandibular prostheses because of oral cancer. In addition, glossectomy patients experience a decrease in swallowing function. Therefore, it is sometimes difficult to spit saliva using this device. 

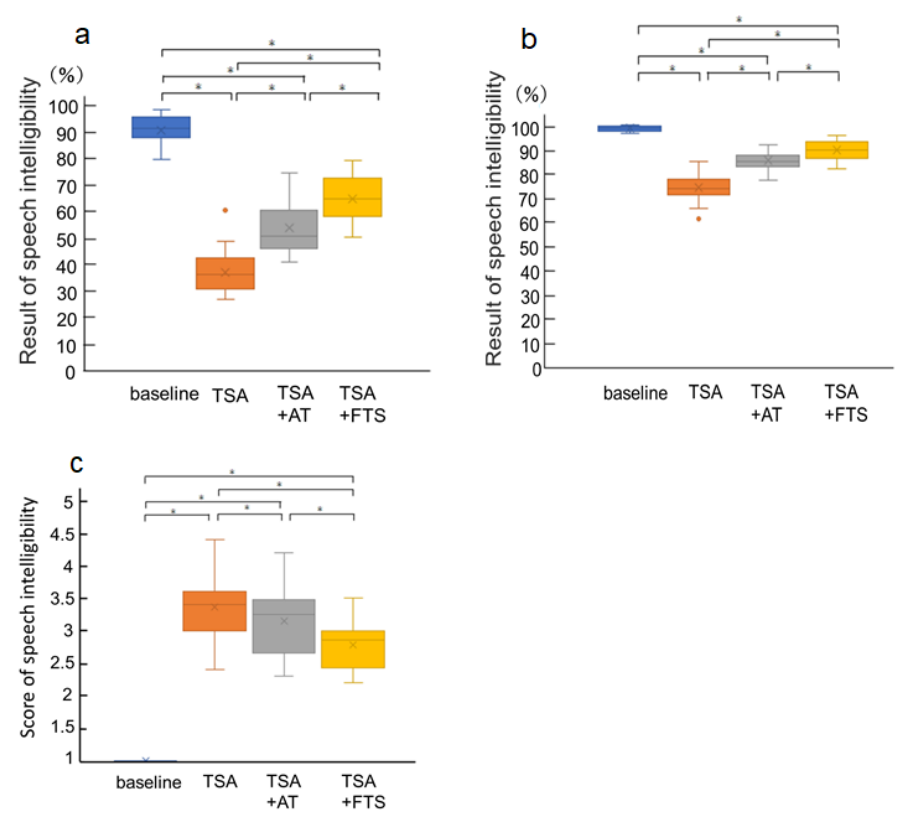

Fig. 5. Mean scores of speech intelligibility. a: 100 Japanese monosyllables; b: 40 Japanese words; c: short story titled "The North Wind and the Sun." Mean value of the first and second measured results with FTS/AT was used as representative value, respectively. Only TSA and no appliance were measured only once.

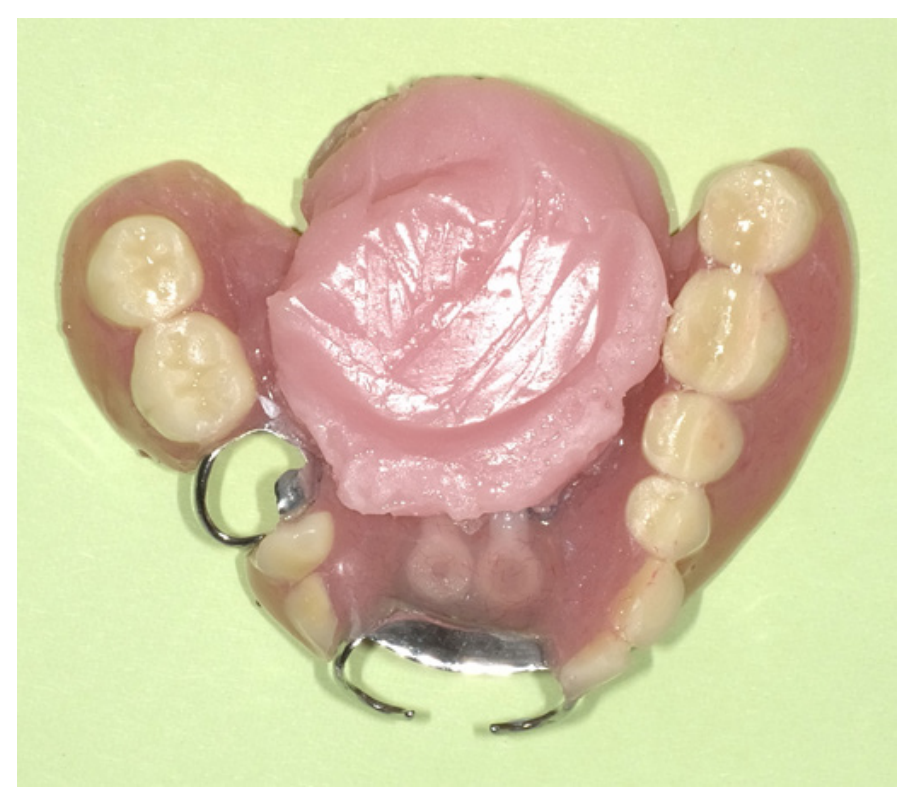

Fig. 6. Photograph of a patient with flat surface tongue prosthesis as an example photograph of clinical application. The devices were fixed at both the upper and lower dental arches.

Participants did not wear PAP with TSA + AT in this study, although they did with TSA + FTS. Wearing PAP with TSA+AT significantly decreased the volume of the oral cavity, which resulted in lower speech intelligibility than without PAP. Glossectomy patients with artificial tongues usually did not wear PAP in a previous case report [8], which is consistent with our study design.

Glossectomy is a surgical procedure widely used to treat patients with tongue cancer. Prosthetic treatment is a functional rehabilitation method for speech disorders after tongue resection. When the extent of tongue resection is small, PAP can help patients recover their speech function. Conversely, when a large part of the tongue or the whole tongue is resected, PAP cannot make contact with the residual tongue, which leads to a significant decline in speech function. Kimata et al. reported that reconstruction of the tongue using a microsurgical technique contributed to the improvement of speech function [17]. However, it is still difficult for patients with extensive tongue defects to recover speech function using this technique.

A systematic review of total glossectomy patients reported that mean single-word intelligibility and mean sentence intelligibility at 12 months post-surgery were $44 \%$ and $66 \%$, respectively [18]. Although the level of evidence in this systematic review was not high due to the small number of participants in the studies on which this review was based, our results were remarkably favorable. In addition, Dziegielewskia et al. reported mean sentence intelligibility of $75 \%$ and single word intelligibility of $62 \%$ for total glossectomy patients at 12 months postoperatively [19]. They concluded that the treatment outcome of speech intelligibility was excellent compared to previous reports, which is similar to the results in this study.

Matsui et al. performed a multicenter study on the recovery of speech function after tongue reconstructive surgery in cancer patients, and the speech intelligibility test was conducted using 100 Japanese monosyllables in a group of patients who had undergone unilateral excision of the subtotal tongue to a depth of more than two-thirds midline in the anterior floor of the oral cavity [20]. Following resection, the state of the remaining movable part of the tongue was presumed to be similar to the state in which tongue movement was restricted using TSA in this study. In the patient group, the speech intelligibility test result after reconstructive surgery was $41.5 \pm 20.2 \%$ [20]. Conversely, the test result when TSA was used for tongue movement suppression was $36.8 \pm 8.0 \%$. Additionally, the speech intelligibility score increased to $64.5 \pm 8.2 \%$ when the test was performed with FTS. The score for the test with 40 Japanese words, using FTS, was $89.5 \%$ on average, indicating high functional recovery. The speech intelligibility test score in this study was much higher than that reported by Matsui et al., which indicated that plastic surgery and prosthodontic treatment complement each other.

In a previous clinical trial [21], speech intelligibility for healthy adults with dentition $(59 \pm 12$ years) was $69.79 \pm 10.60 \%$. In addition, speech intelligibility for newly adopted removable maxillary denture wearers was $66.93 \pm 9.21 \%$. These results suggest that speech intelligibility with TSA + FTS is sufficient for speech improvement. On the other hand, there are several types of dysarthia, including flaccid, ataxic, and hypokinetic. In a clinical trial by Patel et al., speech intelligibility for patients with dysarthia varied widely from $6.4 \%$ to $53.6 \%$ [22]. Although it depends on the severity of the underlying disease, speech intelligibility in these patients is low in most cases.

Five examiners $(29.0 \pm 2.1$ years $)$ without hearing disorder participated in this study to evaluate speech intelligibility, and they did not perform calibration regarding speech intelligibility test. The lack of calibration among examiners might have an effect on the validity and/or reliability of the examiners' evaluations. However, we adopted the same methodology described by Suwaki et al. [23]. to evaluate the speech intelligibility of the participants. There were no significant differences among examiners in the evaluation of speech intelligibility. The population of examiners and participants in this study was almost the same as that of Suwaki et al. In addition, a previous report suggested a difference between younger and older listeners in the evaluation of specific speaker characteristics [24]. Thus, we thought that the setting of examiners was appropriate, regardless of whether they were not calibrated.

\subsection{Clinical applicability}

In our study, the artificial tongue was designed with a flat surface that could be brought into contact with the PAP to regulate the flow path of exhalation. The improvement in speech function shown in this study can be reproduced clinically using a flat surface that is capable of blocking exhalation at the anterior part of the oral cavity, relative to the upper and lower devices. Figure 6 shows a photograph of a patient wearing a tongue prosthesis in our outpatient clinic. As seen in Fig. 6, in cases with several teeth remaining in the mandible, it is possible to create a stable articulation point in the anterior part of the oral cavity with a fixed mandibular device, 
which explains the improvement in speech function observed in this study. The patient shown in Figure 6 wore a traditional prosthesis after tongue resection, which made communication difficult; however, using this artificial tongue, he could comfortably converse over the phone.

\subsection{Limitation}

Our study has some limitations. First, only healthy participants were allowed to participate in the study. Although the movement at the anterior part of the tongue is completely suppressed by TSA, contact between the soft palate and the posterior part of the tongue cannot be suppressed. Therefore, the oral function of patients with tongue resection at the posterior part of the tongue is not accurately recreated, indicating that other functions might play an alternative role in pronunciation. However, none of the participants in this study underwent any resection in the posterior part of the tongue.

Second, the participants used the artificial tongue only for a short term in this study; therefore, the long-term effects of wearing an artificial tongue are unclear. It is believed that there is a difference in the capacity to adapt to the use of tongue prostheses in clinical practice. Moreover, the artificial tongue used in this study had a standard size and shape. If an expert could have constructed an artificial tongue with an anatomical shape for our study, we might have observed a better improvement in speech function. The small number of medical institutions that have the capacity to make a large number of tongue prostheses is another limitation for the application of anatomical artificial tongues. Thus, it is necessary to create a universal method to build an artificial tongue with an anatomical form that can achieve better oral functions in the future.

Finally, we could not evaluate swallowing function, although speech improvement using this device was shown. Patients undergoing glossectomy often have speech disorders as well as dysphagia. In addition, the management of saliva using this device is unclear. Therefore, future studies are needed to evaluate whether swallowing function using this device is improved.

\section{Conclusion}

Our results suggest that PAP with flat surfaces and an artificial tongue can improve speech function. This structure can be easily applied in cases where traditional artificial tongue are applicable, regardless of the variation in the remaining palate, tongue, or floor of the mouth; thus, making it a promising and broadly applicable treatment option.

\section{Acknowledgements}

The authors would like to express their sincere gratitude to Dr. Masaaki Sato for his help in conducting this study.

\section{Funding}

None.

\section{Conflict of interests}

The authors declare no conflicts of interest associated with this study.

\section{References}

[1] Neville BW. Oral cancer and precancerous lesions. CA Cancer J Clin. 2002;52:195-215.

[2] Anna Karinne Costa Bandeira. Elma H. M. Azevedo. Jose' Guilherme Vartanian. Ine^s N. Nishimoto. Luiz Paulo Kowalski. Elisabete Carrara-de Angelis. Quality of Life Related to Swallowing After Tongue Cancer Treatment. Dysphagia. 2008 23(2):183-92.
[3] Lisette van der Molen. Maya A, van Rossum. Lori M. Burkhead. Ludi E. Smeele. Frans J. M. Hilgers. Functional outcomes and rehabilitation strategies in patients treated with chemoradiotherapy for advanced head and neck cancer: a systematic review. Eur Arch Otorhinolaryngol. 2009 266(6):889-900.

[4] Fang QG, Shi S, Zhang X, Li ZN, Liu FY, Sun CF. Assessment of the quality of life of patients with oral cancer after pectoralis major myocutaneous flap reconstruction with a focus on speech. J Oral Maxillofac Surg. 2013;71(11):2004.e1-5.

[5] Jien Morimata. Takafumi Otomaru. Mai Murase. Mihoko Haraguchi. Yuka Sumita. Hisashi Taniguchi. Investigation of factor affecting health-related quality of life in head and neck cancer patients. Gerodontology. 2013;30:194200.

[6] Oscar J Manrique. Hyuma A Leland. Claude-Jean Langevin. Alex Wong. Joseph N Carey. Pedro Ciudad. Hung-Chi Chen. Ketan M Patel. Optimizing Outcomes following Total and Subtotal Tongue Reconstruction: A Systematic Review of the Contemporary Literature. J Reconstr Microsurg. 2017;33(2):103-111.

[7] de Carvalho V, Sennes LU. Speech and swallowing data in individual patients who underwent glossectomy after prosthetic rehabilitation. Int J Dent. 2016;2016:6548014. doi:10.1155/2016/6548014.

[8] Wheeler RL, Logemann JA. Maxillary reshaping prostheses: effectiveness in improving speech and swallowing of postsurgical oral cancer patients. J Prosthet Dent. 1980;43:313-9.

[9] Furia CL, Kowalski LP, Latorre MR, Angelis EC, Martins NM, Barros AP et al. Speech intelligibility after glossectomy and speech rehabilitation. Arch Otolaryngol Head Neck Surg. 2001;127(7):877-83.

[10] Leonard R, Gillis R. Effects of a prosthetic tongue on vowel intelligibility and food management in a patient with total glossectomy. J Speech Hear Disord. 1982:47(1):25-30.

[11] H.S. COTERT, E. ARAS. Mastication, deglutition and speech considerations in prosthodontic rehabilitation of a total glossectomy patient. J Oral Rehabilitation 1999 26; 75-79.

[12] Amir A. Sabouri, Anahita Safari, Jafar Gharechahi, Samira Esmailzadeh, Prosthodontic Rehabilitation for Total Glossectomy with a Magnetic Detachable Mandibular Tongue Prosthesis: A Clinical Report. J of Prosthodonti cs. $2012, ; 21: 404-407$

[13] Kozaki K, Kawakami S, Konishi T, Ohta K, Yano J, Onoda T, et al. Structure of a new palatal plate and the artificial tongue for articulation disorder in a patient with subtotal glossectomy. Acta Med Okayama. 2016;70:205-11.

[14] Balasubramaniam MK, Chidambaranathan AS, Shanmugam G, Tah R. Rehabilitation of glossectomy cases with tongue prosthesis: a literature review. J CLIN Diagn RES. 2016;10(2):1-4.

[15] Umino S, Masuda G, Ono S, Fujita K. Speech intelligibility following maxillectomy with and without a prosthesis: an analysis of 54 cases. J Oral Rehabil. 1998:25:153-8.

[16] Johansson L, Möller S, Olofsson K, Linder J, Nordh E, Blomstedt P, et al. Word-level intelligibility after caudal zona incerta stimulation for Parkinson's disease. Acta Neurol Scand. 2014;130(1):27-33.

[17] Ono T, Hamamura M, Honda K, Nokubi T. Collaboration of a dentist and speech-language pathologist in the rehabilitation of a stroke patient with dysarthria: a case study. Gerodontology. 2005;22:116-9.

[18] Kimata Y, Matsumoto H, Sugiyama N, Onoda S, Sakuraba M. Lessons learned from unfavorable microsurgical head and neck reconstruction: Japan National Cancer Center Hospital and Okayama University Hospital. Clin Plast Surg. 2016;43(4):729-37.

[19] Peter T. Dziegielewski, Michael L. Ho; Jana Rieger, Prabhjyot Singh, Morgan Langille, Jeffrey R. Harris, Hadi Seikaly. Total Glossectomy With Laryngeal Preservation and Free Flap Reconstruction: Objective Functional Outcomes and Systematic Review of the Literature. The Laryngoscope. 2013;123:140-145

[20] Dziegielewski PT, Rieger J, Shama MA, O'Connell DA, Harris JR, Seikaly. Beavertail modification of the radial forearm free flap in total oral glossectomy reconstruction: Technique and functional outcomes. Oral Oncol. 2019;96:71-76.

[21] Matsui Y, Ohno K, Yamashita Y, Takahashi K. Factors influencing postoperative speech function of tongue cancer patients following reconstruction with fasciocutaneous/ myocutaneous flaps - a multicenter study. Int J Oral Maxillofac Surg. 2007;36:601-9.

[22] Florian Stelzle, Max Riemann, Alfred Klein, Nicolai Oetter, Maximilian Rohde, Andreas Maier, Stephan Eitner, Friedrich Wilhelm Neukam, Christian Knipfer.Long-term Outcome of Speech Intelligibility in Maxillary Dental Rehabilitation with Full Dentures: A Prospective Study Using Automatic Speech Quantification. Speech Intelligibility in Maxillary Dental Rehabilitation. 2017:30:419-425.

[23] Rupal Patel, Nicole Usher, Heather Kember, Scott Russell, Jacqueline LauresGore. The influence of speaker and listener variables on intelligibility of dysarthric speech. Journal of Communication Disorders. 2014;51:13-18.

[24] Suwaki M, Nanba K, Ito E, Kumakura I, Minagi S. The effect of nasal speaking valve on the speech under experimental velopharyngeal incompetence condition. J Oral Rehabil. 2008 May;35(5):361-9.

[25] Huiwen Goy, M. Kathleen Pichora-Fuller, Pascal van Lieshout. Effects of age on speech and voice quality ratings. Journal of the Acoustical Society of America. 2016;139(4):1648-1659.

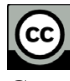

Copyright: This is an open-access article distributed under the terms of Creative Commons Attribution License 4.0 (CCBY 4.0), which allows users to distribute and copy the material in any format so long as attribution is given to the author(s). 